KYUNGPOOK Math. J. 52(2012), 33-38

http://dx.doi.org/10.5666/KMJ.2012.52.1.33

\title{
On a Certain Integral Operator
}

SAURABH PorWaL*

Department of Mathematics, U. I. E. T. Campus, C. S. J. M. University, Kanpur208024, (U. P.), India

e-mail : saurabhjcb@rediffmail.com

Muhammed Kamal Aouf

Department of Mathematics, Faculty of Science, University of Mansoura 35516, Egypt

e-mail : mkaouf127@yahoo.com

ABstract. The purpose of the present paper is to investigate mapping properties of an integral operator in which we show that the function $g$ defined by

$$
g(z)=\left\{\frac{c+\alpha}{z^{c}} \int_{0}^{z} t^{c-1}\left(D^{n} f\right)^{\alpha}(t) d t\right\}^{1 / \alpha} .
$$

belongs to the class $S(A, B)$ if $f \in S(n, A, B)$.

\section{Introduction}

Let $A$ denote the class of functions of the form

$$
f(z)=z+\sum_{k=2}^{\infty} a_{k} z^{k}
$$

which are analytic in the open unit disc $U=\{z:|z|<1\}$. Further $S$ denotes the subclass of $A$ consisting of functions $f(z)$ of the form (1.1) which are univalent in $U$. For the functions $f$ and $g$ in $A$, we say that $f$ is subordinate to $g$ in $U$, and write $f \prec g$, if there exists a Schwarz function $w(z)$ in $A$ with $\omega(0)=0$ and $|\omega(z)|<1$ such that $f(z)=g(\omega(z))$ in $U$, (see $[16])$.

Now for $n \in N_{0},-1 \leq A<B \leq 1$ and $z \in U$, suppose that $S(n, A, B)$ denote the family of functions of the form (1.1) which satisfy the condition

$$
\frac{D^{n+1} f(z)}{D^{n} f(z)} \prec \frac{1+A z}{1+B z},
$$

* Corresponding Author.

Received February 9, 2011; accepted September 23, 2011.

2010 Mathematics Subject Classification: 30C45, 30C80.

Key words and phrases: Analytic, Univalent, Subordination, Integral Operator.

The present investigation was supported by the University Grant Commission under grant No. 11-12/2006(SA-I). 
where $D^{n}$ stands for the Salagean operator introduced by Salagean in [19]. For $n=0$, we denote the class $S(n, A, B)$ by $S(A, B)$.

By specializing the parameters in subclass $S(n, A, B)$, we obtain the following known subclasses studied earlier by various researchers.

(i) If we put $A=-(1-2 \beta), 0 \leq \beta<1, B=1$ then it reduces to the class $S(n, \beta)$ studied by Kadioğlu [12].

(ii) If we put $n=0, A=-(1-2 \beta), 0 \leq \beta<1, B=1$ then it reduces to the class $S^{*}(\beta)$ of univalent starlike functions of order $\beta$, studied by Robertson [18] and Silverman $[20]$.

(iii) If we put $n=1, A=-(1-2 \beta), 0 \leq \beta<1, B=1$ then it reduces to the class $K(\beta)$ of univalent convex functions of order $\beta$, studied by Robertson [18] and Silverman $[20]$.

Now, we introduce a new integral operator $g: A \rightarrow A$ as follows

$$
g(z)=\left\{\frac{c+\alpha}{z^{c}} \int_{0}^{z} t^{c-1}\left(D^{n} f\right)^{\alpha}(t) d t\right\}^{1 / \alpha},
$$

where $n \in N_{0}, \alpha>0, c>-\alpha$.

The study of the above integral operator is of special interest because it reduces to various well-known integral operators such as Alexander integral operator [3], Libera integral operator [14], Bernardi integral operator [4] etc. for different choices of $n$ and $\alpha$.

Several authors such as $([1],[2],[5],[6],[7],[8],[9],[13],[17])$ studied the interesting properties of the various integral operators. In the present paper, by employing a different technique we obtain condition, if $f \in S(n, A, B)$ then $g \in$ $S(A, B)$.

\section{Main results}

To establish our main result we require the following lemmas.

Lemma 2.1. A function $f$ of the form (1.1) belongs to $S(n, A, B),-1 \leq A<B \leq$ 1 , if and only if

$$
\left|\frac{D^{n+1} f(z)}{D^{n} f(z)}-m\right|<M, \quad z \in U,
$$

where

$$
m=\frac{1-A B}{1-B^{2}} \quad \text { and } \quad M=\frac{B-A}{1-B^{2}} .
$$

Proof. Let $f \in S(n, A, B)$. For a Schwarz function $\omega(z)$ in $A$ with $\omega(0)=$ 0 and $|\omega(z)|<1$ the condition (1.2) is equivalent to

$$
\frac{D^{n+1} f(z)}{D^{n} f(z)}=\frac{1+A \omega(z)}{1+B \omega(z)}
$$


or

$$
\begin{aligned}
\frac{D^{n+1} f(z)}{D^{n} f(z)}-m= & \frac{(1-m)+(A-B m) \omega(z)}{1+B \omega(z)} \\
& =M h(z),
\end{aligned}
$$

where $h(z)=-\frac{(B+\omega(z))}{1+B \omega(z)}$. Since $|h(z)|<1$, the inequality (2.1) immediately follows from (2.4).

Conversely, let $f$ satisfy (2.1). Then

$$
\left|\frac{D^{n+1} f(z)}{M D^{n} f(z)}-\frac{m}{M}\right|<1, \quad z \in U .
$$

Let

$$
q(z)=\frac{D^{n+1} f(z)}{M D^{n} f(z)}-\frac{m}{M}
$$

and we define

$$
\omega(z)=\frac{q(0)-q(z)}{1-q(0) q(z)} .
$$

Clearly the function $\omega(z)$ is analytic in $U$, and satisfies $\omega(0)=0$ and $|\omega(z)|<1$ for $z \in U$. Since $q(0)=-B$, from $(2.6)$ we have

$$
q(z)=-\frac{(B+\omega(z))}{1+B \omega(z)} .
$$

Eliminating $q(z)$ from (2.5) and (2.7), we obtain (2.3). Hence $f \in S(n, A, B)$.

The next lemma is due to Jack [11].

Lemma 2.2. If the function $\omega(z)$ is analytic for $|z| \leq r<1, \omega(0)=$ 0 and $\left|\omega\left(z_{0}\right)\right|=\max _{|z|=r}|\omega(z)|$ then $z_{0} \omega^{\prime}\left(z_{0}\right)=k \omega\left(z_{0}\right)$, where $k$ is a real number such that $k \geq 1$.

Theorem 2.1. If $f \in S(n, A, B)$ and $g$ is defined by (1.3), where $\alpha$ and $c$ are real numbers such that $\alpha>0, n \in N_{0}$ and $c \geq \frac{-\alpha(1+A)}{1+B}$. Then the function $g$ belongs to $S(A, B)$. In (1.3) powers denote principal ones.

Proof. Let us define a function $\omega(z)$ such that

$$
\omega(z)=\frac{\frac{z g^{\prime}(z)}{g(z)}-1}{A-B \frac{z g^{\prime}(z)}{g(z)}} .
$$

So that

$$
\frac{z g^{\prime}(z)}{g(z)}=\frac{1+A \omega(z)}{1+B \omega(z)}
$$


where $\omega(z)$ is either analytic or meromorphic in $U$. Clearly $\omega(0)=0$ we claim that $\omega(z)$ is analytic in $U$, and $|\omega(z)|<1$ for $z \in U$, which we will prove by contradiction.

From (1.3) and (2.8), we have

$$
(c+\alpha)\left\{\frac{D^{n} f(z)}{g(z)}\right\}^{\alpha}=\frac{(c+\alpha)+(A \alpha+B C) \omega(z)}{1+B \omega(z)} .
$$

Logarithmic differentiation of (2.9) with respect to $z$ yields (2.10)

$\frac{D^{n+1} f(z)}{D^{n} f(z)}-m=\frac{(1-m)+(A-B m) \omega(z)}{1+B \omega(z)}-\frac{(B-A) z \omega^{\prime}(z)}{\{1+B \omega(z)\}\{(c+\alpha)+(A \alpha+B C) \omega(z)\}}$.

Let $r^{*}$ be the distance, from the origin, of the pole of $\omega(z)$ nearest the origin. Then $\omega(z)$ is analytic in $|z|<r_{0}=\min \left\{r^{*}, 1\right\}$. By Lemma 2.2, for $|z| \leq r,\left(r \leq r_{0}\right)$, there exists a point $z_{0}$ such that,

$$
z_{0} \omega^{\prime}\left(z_{0}\right)=k \omega\left(z_{0}\right), \quad k \geq 1
$$

From (2.10) and (2.11), we have

$$
\frac{D^{n+1} f\left(z_{0}\right)}{D^{n} f\left(z_{0}\right)}-m=\frac{N\left(z_{0}\right)}{D\left(z_{0}\right)},
$$

where $N\left(z_{0}\right)=(1-m)(c+\alpha)+\{(c+\alpha)(A-B m)+(A \alpha+B C)(1-m)-k(B-$ A) $\} \omega\left(z_{0}\right)+\{(A \alpha+B C)(A-B m)\} \omega^{2}\left(z_{0}\right)$ and

$$
D\left(z_{0}\right)=(c+\alpha)+(A \alpha+2 B C+B \alpha) \omega\left(z_{0}\right)+B(A \alpha+B C) \omega^{2}\left(z_{0}\right) .
$$

Now suppose that it were possible to have $\max _{|z|=r}|\omega(z)|=\left|\omega\left(z_{0}\right)\right|=1$ for some $r$, $r<r_{0} \leq 1$. Then by using the identities $A-B m=-M$ and $B-A=\frac{\left(M^{2}-(m-1)^{2}\right)}{M}$, we have

$$
\left|N\left(z_{0}\right)\right|^{2}-M^{2}\left|D\left(z_{0}\right)\right|^{2}=a+2 b \operatorname{Re}\left\{\omega\left(z_{0}\right)\right\},
$$

where

$$
a=k(B-A)\{k(B-A)+2 M(c+\alpha)+2 M B(A \alpha+B C)\},
$$

and

$$
b=k(B-A) M\{(A \alpha+B C)+B(c+\alpha)\} .
$$

From (2.13) we have

$$
\left|N\left(z_{0}\right)\right|^{2}-M^{2}\left|D\left(z_{0}\right)\right|^{2}>0,
$$

provided $a \pm 2 b>0$. 
Now $a+2 b=k(B-A)[k(B-A)+2 M(1+B)\{c(1+B)+\alpha(1+A)\}]>0$, provided $c \geq \frac{-\alpha(1+A)}{(1+B)}$, and

$$
a-2 b=k(B-A)[k(B-A)+2 M(1-B)\{c(1-B)+\alpha(1-A)\}]
$$

$>0$, provided $c \geq \frac{-\alpha(1-A)}{(1-B)}$.

Thus from (2.12) and (2.14), we have

$$
\left|\frac{D^{n+1} f\left(z_{0}\right)}{D^{n} f\left(z_{0}\right)}-m\right|>M
$$

provided $c \geq \max .\left\{\frac{-\alpha(1+A)}{(1+B)}, \frac{-\alpha(1-A)}{(1-B)}\right\}=\frac{-\alpha(1+A)}{(1+B)}$.

But this is, in view of Lemma 2.1, contrary to our assumption $f \in S(n, A, B)$. Therefore, we can not have $|\omega(z)|=1$ in $|z|<r_{0}$. Since $|\omega(0)|=0,|\omega(z)|$ is continuous and $|\omega(z)| \neq 1$ in $|z|<r_{0}, \omega(z)$ can not have a pole at $|z|=r_{0}$. Since $r_{0}$ is arbitrary, we conclude that $\omega(z)$ is analytic in $U$, and satisfies $|\omega(z)|<1$ for $z \in U$.

Hence, from (2.8), $g \in S(A, B)$.

Remark 2.1. If we put $A=-(1-2 \beta)$, where $0 \leq \beta<1, B=1$ and $n=0$ then the class $S(n, A, B)$ reduces to the well-known class $S^{*}(\beta)$ of univalent starlike functions of order $\beta$ and Theorem 2.1 reduces as

Corollary 2.1. Let $\alpha$ and $c$ be real numbers such that $\alpha>0$ and $c \geq-\alpha \beta$. If $f \in S^{*}(\beta)$, then the function $g$ defined by

$$
g(z)=\left\{\frac{c+\alpha}{z^{c}} \int_{0}^{z} t^{c-1} f^{\alpha}(t) d t\right\}^{1 / \alpha}
$$

is also in the class $S^{*}(\beta)$.

Remark 2.2. The above result is also obtained by Gupta and Jain [10] only for the case when $\alpha$ and $c$ are positive integer.

Remark 2.3. If we put $\beta=0$ then we obtain the corresponding result of Miller et al. [15].

Acknowledgements The first author is thankful to Prof. K. K. Dixit, Department of Mathematics, Gwalior Institute of Information Technology, Gwalior, (M. P.) for their encouragement.

\section{References}

[1] A. M. Acu, Some preserving properties of the generalized Alexander operator, General Math., 10(3-4)(2002), 37-46. 
[2] A. M. Acu and E. Constantinescui, Some preserving properties of a integral operator, General Math., 15(2-3)(2007), 9-15.

[3] J. W. Alexander, Functions which map the interior of the unit circle upon simple regions, Ann. of Math., 17(1915-1916), 12-22.

[4] S. D. Bernardi, Convex and starlike univalent functions, Trans. Amer. Math. Soc., 135(1969), 429-446.

[5] D. Blezu, On univalence criteria, General Math., 14(1)(2006), 87-93.

[6] D. Breaz, Univalence properties for a general integral operator, Bull. Korean Math. Soc., 46(3)(2009), 439-446.

[7] D. Breaz, A convexity property for an integral operator on the class $S_{P}(\beta)$, J. Ineq. Appl., (2008), Art. ID-143869, 1-4.

[8] D. Breaz, An integral univalent operator of the class $S(P)$ and $T_{2}$, Novi Sad. J. Math., 37(1)(2007), 9-15.

[9] N. Breaz and D. Breaz, Sufficient univalent conditions for an integral operator, Proc. Int. Symp. New Devp. GFTA, (2008), 59-63.

[10] V. P. Gupta and P. K. Jain, On starlike functions, Rend. Math., 9(1976), 433-437.

[11] I. S. Jack, Functions starlike and convex of order $\alpha$, J. London Math. Soc., 2(3)(1971), 469-474.

[12] E. Kadioğlu, On subclass of univalent functions with negative coefficients, Appl. Math. Comput., 146(2003), 351-358.

[13] Jian Lin Li, Some properties of two integral operators, Soochow J. Math., 25(1)(1999), 91-96.

[14] R. J. Libera, Some classes of regular univalent functions, Proc. Amer. Math. Soc., 16(1965), 755-758.

[15] S. S. Miller, P. T. Mocanu and M. O. Reade, Starlike integral operators, Pacific J. Math., 79(1978), 157-168.

[16] Z. Nehari, Conformal Mapping, Mc-Graw Hill, New York, (1952).

[17] G. I. Oras, A univalence preserving integral operator, J. Inequal. Appl., (2008), Art. ID-263408, 1-10.

[18] M. S. Robertson, On the theory of univalent functions, Ann. Math., 37(1936), 374 408.

[19] G. S. Salagean, Subclasses of univalent functions, Complex Analysis-Fifth Romanian Finish Seminar, Bucharest, 1(1983), 362-372.

[20] H. Silverman, Univalent functions with negative coefficients, Proc. Amer. Math. Soc., 51(1975), 109-116. 